\title{
Article
}

\section{Well-posedness for a modified nonlinear Schrödinger equation modeling the formation of rogue waves}

\author{
Curtis Holliman ${ }^{1, *}$ and Logan Hyslop ${ }^{1}$ \\ 1 Department of Mathematics, The Catholic University of America, Washington, DC 20064, USA. \\ * Correspondence: holliman@cua.edu
}

Academic Editor: Michael Th. Rassias

Received: 10 March 2021; Accepted: 19 June 2021; Published: 30 June 2021.

\begin{abstract}
The Cauchy problem for a higher order modification of the nonlinear Schrödinger equation (MNLS) on the line is shown to be well-posed in Sobolev spaces with exponent $s>\frac{1}{4}$. This result is achieved by demonstrating that the associated integral operator is a contraction on a Bourgain space that has been adapted to the particular linear symbol present in the equation. The contraction is proved by using microlocal analysis and a trilinear estimate that is shown via the $[k ; Z]$-multiplier norm method developed by Terence Tao.
\end{abstract}

Keywords: Modified nonlinear Schrödinger equation; Well-posedness in Sobolev spaces; Rogue waves; Initial value problem; Dispersive equations.

MSC: 35Q53; 37K10.

\section{Introduction}

$\mathbf{W}$

e consider the initial value problem (ivp) for a particular modification of nonlinear Schrödinger (MNLS) equation that has been used to model the formation of rogue or freak waves [1]. The Cauchy problem for the MNLS is given by

$$
\left\{\begin{array}{l}
u_{t}+i u_{x x}-u_{x x x}+i|u|^{2} u+|u|^{2} u_{x}+u^{2} \bar{u}_{x}+i u \partial_{|x|}\left(|u|^{2}\right)=0 \\
u(x, 0)=\varphi(x), \quad x, t \in \mathbb{R} .
\end{array}\right.
$$

We use the symbol $\partial_{|x|}$, or partial with respect to $|x|$, which appears in the last nonlinear term to represent the Riesz derivative which can be described as the Fourier multiplier

$$
\widehat{f_{|x|}}(\xi)=-|\xi| \widehat{f}(\xi)
$$

The Riesz derivative is of particular interest in this equation and it appears in many applications of fractional calculus. It represents a fractional derivative term that appears in many equations, including the (1), Benjamin-Ono and the fractional Schrödinger equations. Recent scattering results for the scattering results for related Dysthe equation, which has many structural similarities to the MNLS with the Riesz derivative occuring in the nonlinearities, can be found in [2].

Rogue waves can be informally thought of as extremely rare events arising from the constructive interference of the surrounding waves. This construction interference can result in waves with heights as large as eight times that of the surrounding free surface [3,4]. There are many real world consequences of these large spontaneous events, which can include damage and disruption to commercial shipping, human lives and many other nautical operations. For an examination of the mechanisms that lead to the development of these rogue waves, we refer the readers to [5-8], and the references found therein.

In this paper, we consider a higher-order approximation of the fully nonlinear model, which is given by

$$
u_{t}+\frac{1}{2} u_{x}+\frac{i}{8} u_{x x}-\frac{1}{16} u_{x x x}+\frac{i}{2}|u|^{2} u+\frac{3}{2}|u|^{2} u_{x}+\frac{1}{4} u^{2} \bar{u}_{x}+i u \partial_{|x|}\left(|u|^{2}\right)=0 .
$$


Changing variables and using a Galilean lift simplifies (3) to the equation we refer to as the MNLS (1). This particular approximation has been used in applied settings $[9,10]$, and there are other modification to the NLS which have been studied, including including the compact Zakharov equation [11,12].

The basis of the analytical investigation in this work work lies in the theory of the nonlinear Schrödinger equation (NLS),

$$
i u_{t}+u_{x x}+|u|^{2} u=0
$$

and the Korteweg-de Vries equation (KdV),

$$
u_{t}+u u_{x}+u_{x x x}=0 .
$$

The inspiration for this work comes from the seminal works of Bourgain $[13,14]$, where he proves that the NLS and KdV equations are, among other things, well-posed. In particular, as the method of proof largely follows the same principles for both of these equations, we were interested in adapting Bourgain's method, as further extended in [15] and [16]. Here we adapt these methods to an equation where the linear symbol was a combination of both that of the NLS and KdV. In a similar vein, this work builds on previous analysis of a modified Korteweg-de Vries ( $\mathrm{KdVm}$ ) equation where the dispersion was taken to be an odd natural number $m \geq 3$ and is given by

$$
\partial_{t} u+\partial_{x}^{m} u+u \partial_{x} u=0
$$

In [17] and [18], the well-posedness for the KdVm was demonstrated for initial data in Sobolev spaces $H^{s}$ where $s \geq-(m-1) / 4$. Thus, we immediately see that there is a relationship between the structure of the linear symbol and the smoothness required of the initial data.

The broad strategy to demonstrate well-posedness of (1) relies on using a contraction mapping argument on a Bourgain space $X_{s, b}$. Here the Bourgain space will be tailored to the linear symbol present in (1). Additionally, the presence of the Riesz derivative creates some analytical problems to overcome in the trilinear estimates. There is a significant amount of literature devoted to results on the NLS, and we refer the reader to the following works and the references therein, [9,10,15,19-25].

Our work revolves around reformulating (1) into a related fixed point problem for an integral operator $T$. We start by defining $W$ to be the solution operator to the homogeneous problem associated with (1),

$$
W(t) \varphi(x)=\frac{1}{2 \pi} \int_{\mathbb{R}} e^{x i \xi+i t \gamma(\xi)} \widehat{\varphi}^{x}(\xi) d \xi,
$$

where $\gamma(\xi)=\xi^{2}-\xi^{3}$. The associated Bourgain space to our problem is given by:

Definition 1. Let $X_{s, b}$ be a space such that, for any $s, b \in \mathbb{R}, X_{s, b}$ is the completion of the Schwartz space $\mathcal{S}\left(\mathbb{R}^{2}\right)$ with respect to the norm

$$
\||u|\|_{s, b}=\left(\iint_{\mathbb{R}^{2}}\langle\xi\rangle^{2 s}\langle\tau-\gamma(\xi)\rangle^{2 b}|\widehat{u}(\xi, \tau)|^{2} d \xi d \tau\right)^{\frac{1}{2}}
$$

where $\langle\xi\rangle=1+|\xi|$.

Using the usual methods, we reformulate the problem in terms of the following integral operator. Should the reader desire more details on going about this procedure, we refer them to [17,18]. Taking the space-time Fourier transform of $w$ allows us to express the mapping $T$ in the phase space $(\xi, \tau)$. To continue, we use a cutoff function, $\psi \in C_{0}^{\infty}(\mathbb{R})$ with $\sup p(\psi) \subset[-1,1]$ and $\psi \equiv 1$ on $[-1 / 2,1 / 2]$. The corresponding integral operator we obtain is

$$
T u(x, t)=\psi(t) W(t) \varphi(x)+\frac{i}{4 \pi^{2}} \psi(t) \iint_{\mathbb{R}^{2}} e^{i x \xi+i t \gamma(\xi)} \widehat{w}(\xi, \tau) d \tau d \xi
$$


where $w$ corresponds to the sum of the nonlinearities

$$
w=i|u|^{2} u+|u|^{2} u_{x}+|u|^{2} \bar{u}_{x}+i u \partial_{|x|}\left(|u|^{2}\right) .
$$

In handling $w$, our focus will be on the final term $i u \partial_{|x|}\left(|u|^{2}\right)$. The Riesz derivative makes this term the most novel. For an analysis of this equation where $w$ strictly has the NLS nonlinearity, or $w=i|u|^{2} u$, we refer the reader to [19]. Additionally, we have adjusted our proof to use the strategies developed by Tao in [26] and [27].

Our results regarding (1) can now be formalized in the following theorem:

Theorem 1. If $s>\frac{1}{4}$ then for any $\varphi \in H^{s}$ with sufficiently small norm, the integral equation

$$
u=T u,
$$

where $T$ is defined by (8) has a unique solution in the space $X_{s, b}$ for some $b>1 / 2$. Moreover, the data-to-solution map is Lip-continuous.

This paper is organized as follows: In Section 2, we prove Theorem 1 while assuming the necessary trilinear estimates needed to handle the nonlinear terms, which we catalogue in Theorem 2. In Section 3 we provide the trilinear estimates outlines in Theorem 2, which completes the proof of our main result. We note that in the proof of Theorem 2, we focus on the the estimate for the Riesz derivative term. We then give a description of how to adapt this proof to the other terms.

\section{Proof of Theorem 1}

The main tool used to prove Theorem 1 involves the following trilinear estimates summarized below. For convenience in later computations, we consider nonlinearities that include a Hilbert transform $\mathcal{H}$ and $\partial_{x}$ instead of the Riesz derivative $\partial_{|x|}$.

Theorem 2 (Trilinear Estimates). If $s>\frac{1}{4}$, then

$$
\begin{aligned}
& \left|\left\|u^{2} \bar{u}||_{s, b-1} \lesssim\right\|\|u\|_{s, b}^{3}, \quad u \in X_{s, b},\right. \\
& \left.\left\|\left|\partial_{x}\left(u^{2} \bar{u}\right)\right|\right\|\right|_{s, b-1} \lesssim\|\| u \|_{s, b}^{3}, \quad u \in X_{s, b}, \\
& \left\|\left.\left|\partial_{x}(u \mathcal{H}(u \bar{u}))\right|\right|_{s, b-1} \lesssim\right\|\|u\|_{s, b}^{3}, \quad u \in X_{s, b} \text {, }
\end{aligned}
$$

for some $b>\frac{1}{2}$.

Furthermore, to establish that $T$ is a contraction mapping on a ball in $X_{s, b}$ space, we need the following result:

Proposition 1. If $s>\frac{1}{4}$, there exists some $b>\frac{7}{12}$ and $c=c(\psi)>0$ such that

$$
\|\left.|| T u||\right|_{s, b} \leq c\left(\||| u \mid\|_{s, b}^{3}+\|\varphi\|_{H^{s}}\right), u \in X_{s, b}
$$

and

$$
\left\||| T u-T v\left|\left\|_{s, b} \leq c\left|\left\|u+v\left(\frac{1+i \sqrt{3}}{2}\right)\right\|\left\|_{s, b}\right\|\right| u+v\left(\frac{1-i \sqrt{3}}{2}\right)\right\|\left\|_{s, b}\right\| u-v\right|\right\|_{s, b}, u, v \in X_{s, b} .
$$

Proof of Theorem 1. If $\varphi$ satisfies the smallness condition

$$
\|\varphi\|_{H^{s}} \leq \frac{15}{64 c^{\frac{3}{2}}}
$$

then, for any $u$ in the closed ball

$$
B=\left\{u \in X_{s, b}:\|u\| \|_{s, b} \leq \frac{1}{4 c^{\frac{1}{2}}}\right\}
$$


we have

$$
\|T u\|_{s, b} \leq c\left(\frac{1}{64 c^{\frac{3}{2}}}+\frac{15}{64 c^{\frac{3}{2}}}\right)=\frac{1}{4 c^{\frac{1}{2}}} .
$$

Thus demonstrating that $T$ maps the ball $B$ into itself. From (15), we have, for any $u, v \in B$

$$
\begin{aligned}
& \left\||| T u-T v\left|\left\|_{s, b} \leq c\left(\left\|| | u \left|\| _ { s , b } + | \| v ( \frac { 1 + i \sqrt { 3 } } { 2 } ) \| | _ { s , b } ) \left(\|u\|\left\|_{s, b}+\left|\left\|v\left(\frac{1-i \sqrt{3}}{2}\right)\right\|\right|_{s, b}\right)\|\mid u-v\|_{s, b}\right.\right.\right.\right.\right.\right.\right. \\
& =c\left(|||u|||_{s, b}+2|\| v|||_{s, b}\right)\left(\left\|\left.|| u||\right|_{s, b}+2\left|\|v \mid\|_{s, b}\right)\left|\|u-v \mid\|_{s, b}\right.\right.\right. \\
& \leq c \cdot\left(\frac{9}{16 c}\right) \cdot\|\| u-v \|_{s, b} \\
& =\frac{9}{16}\|u-v\|_{s, b}
\end{aligned}
$$

Which proves that $T$ is a contraction mapping on $B$ which guarantees us in turn gives us a unique fixed point by the Banach Contraction Mapping Theorem.

Proof of Lip-Continuous dependence on initial data. Let $\varphi$ and $\chi$ be in $H^{s}$ both satisfying the smallness conditions

$$
\|\varphi\|_{H^{s}} \leq \frac{15}{64 c^{\frac{3}{2}}} \text { and }\|\chi\|_{H^{s}} \leq \frac{15}{64 c^{\frac{3}{2}}} .
$$

Further let $u$ and $v$ be the solutions corresponding to $\varphi$ and $\chi$ respectively. From here, we will use a subscript on $T$ to denote the dependence on the initial data. In particular, as $u$ and $v$ are fixed points, we have $u=T_{\varphi} u$ and $v=T_{\chi} v$. We then see that

$$
\left\||u-v|||_{s, b}=\right\||| T_{\varphi} u-T_{\chi} v||_{s, b}=\|\left|\psi W(\varphi-\chi)+T_{0} u-T_{0} v\right|||_{s, b} .
$$

From here, a simple application of the triangle inequality in conjunction with Proposition 1 will complete the argument. The first term is a linear operator which we will examine below to estimate (25). In particular we use Lemma 2 to handle $\psi$ to get

$$
\left.\||\psi W(\varphi-\chi)|\|\right|_{s, b} \lesssim\|\varphi-\chi\|_{H^{s}} .
$$

For the second term, we follow the same argument used to obtain (19) which gives us $\|\left|T_{0} u-T_{0} v\right|||_{s, b} \leq$ $\frac{9}{16}|||u-v| \|_{s, b}$. We therefore have for a constant $c$

$$
\||u-v|\|_{s, b} \leq c|| \varphi-\chi\left\|_{H^{s}}+\frac{9}{16}\left|\|u-v \mid\|_{s, b} .\right.\right.
$$

Subtracting $\frac{9}{16}|||u-v| \|_{s, b}$ from both sides of (22) allows to conclude

$$
\|u-v \mid\|_{s, b} \leq \frac{16 c}{7}\|\varphi-\chi\|_{H^{s}}
$$

which completes our proof of the Lip-continuity of the data-to-solution map.

Proof of Proposition 1. Before beginning this proof, we state the following Lemma.

Lemma 1. For $s \geq 0$, and $u \in X_{s, b}$ we define

$$
T(u, \bar{u}, u)(x, t) \doteq \psi(t) W(t) \varphi(x)-\psi(t) \int_{0}^{t} W\left(t-t^{\prime}\right) w_{u \bar{u} u}\left(x, t^{\prime}\right) d t^{\prime},
$$


then there exists a constant $c=c_{\psi}$ such that

$$
\left\|\left|T(u, \bar{u}, u)\|\|_{s, b} \leq c\right|\right\| u\left\|_{s, b}^{3}+c\right\| \varphi \|_{H^{s}} .
$$

To continue, we look at the decompose map of $T$ as defined in (8), by adding and subtracting $\psi(\tau-\gamma(\xi))$ in the integral expression to localize near the singularity $\tau=\gamma(\xi)$. We thus obtain the decomposition

$$
\begin{aligned}
\operatorname{Tu}(x, t)= & \frac{1}{2 \pi} \psi(t) \int_{\mathbb{R}} e^{i x \xi+i t \gamma(\xi)} \widehat{\varphi}^{x}(\xi) d \xi \\
& +\frac{i}{4 \pi^{2}} \psi(t) \iint_{\mathbb{R}^{2}} e^{i(\xi x+\tau t)} \frac{1-\psi(\tau-\gamma(\xi))}{\tau-\gamma(\xi)} \widehat{w}(\xi, \tau) d \tau d \xi \\
& -\frac{i}{4 \pi^{2}} \psi(t) \iint_{\mathbb{R}^{2}} e^{i(\xi x+t \gamma(\xi))} \frac{1-\psi(\tau-\gamma(\xi))}{\tau-\gamma(\xi)} \widehat{w}(\xi, \tau) d \tau d \xi \\
& +\frac{i}{4 \pi^{2}} \psi(t) \iint_{\mathbb{R}^{2}} e^{i(\xi x+t \gamma(\xi))} \frac{\psi(\tau-\gamma(\xi))\left[e^{i t(\tau-\gamma(\xi))}-1\right]}{\tau-\gamma(\xi)} \widehat{w}(\xi, \tau) d \tau d \xi .
\end{aligned}
$$

Estimate of (25). Defining

$$
I(x, t) \doteq \frac{1}{2 \pi} \psi(t) \int_{\mathbb{R}} e^{x i \xi+i t \gamma(\xi)} \widehat{\varphi}^{x}(\xi) d \xi,
$$

we take the Fourier transform of $I$ with respect to $x$ and $t$. The $X_{s, b}$ norm of $I$ can then be directly estimated as

$$
\begin{aligned}
\||| I \mid\|_{s, b} & =\left(\iint_{\mathbb{R}^{2}}\langle\xi\rangle^{2 s}\langle\tau-\gamma(\xi)\rangle^{2 b}\left|\widehat{\psi}^{t}(\tau-\gamma(\xi)) \widehat{\varphi}^{x}(\xi)\right|^{2} d \xi d \tau\right)^{\frac{1}{2}}, \\
& \lesssim\left(\int_{\mathbb{R}}\langle\xi\rangle^{2 s}\left|\widehat{\varphi}^{x}(\xi)\right|^{2} \int_{\mathbb{R}}\langle\tau-\gamma(\xi)\rangle^{2 b}\left|\widehat{\psi}^{t}(\tau-\gamma(\xi))\right|^{2} d \tau d \xi\right)^{\frac{1}{2}} \\
& =c_{\psi}\|\varphi\|_{H^{s}}
\end{aligned}
$$

Estimate of (26). Taking

$$
I(x, t) \doteq \frac{1}{4 \pi^{2}} \int_{\mathbb{R}} e^{i x \xi} \int_{\mathbb{R}} e^{i \tau t} \frac{1-\psi(\tau-\gamma(\xi))}{\tau-\gamma(\xi)} \widehat{w}_{u \bar{u} u}(\xi, \tau) d \tau d \xi,
$$

we have

$$
\text { ||(26)||| }\left.\right|_{s, b}=|||\psi(t) I(x, t)|||_{s, b .} .
$$

To continue, we need the following Lemma:

Lemma 2. There exists a $c=c_{\psi}$ such that

$$
\||\psi(t) I(x, t)|||_{s, b} \leq c_{\psi}|||I(x, t)|||_{s, b} .
$$

Using Lemma 2, we see that taking the space-time Fourier transform of $I$ allows us to compute the $X_{s, b}$ norm of $I$ as

$$
\begin{aligned}
\|I \mid\|_{s, b} & =\left(\iint_{\mathbb{R}^{2}}\langle\xi\rangle^{2 s}\langle\tau-\gamma(\xi)\rangle^{2 b}\left|\frac{1-\psi(\tau-\gamma(\xi))}{\tau-\gamma(\xi)} \widehat{w}_{u \bar{u} u}(\xi, \tau)\right|^{2} d \xi d \tau\right)^{\frac{1}{2}}, \\
& \lesssim\left(\int_{\mathbb{R}}\langle\xi\rangle^{2 s} \int_{\mathbb{R}}\langle\tau-\gamma(\xi)\rangle^{2(b-1)}\left|\widehat{w}_{u \bar{u} u}(\xi, \tau)\right|^{2} d \tau d \xi\right)^{\frac{1}{2}}, \\
& \simeq\left\||| w_{u \bar{u} u} \mid\right\|_{s, b-1} \\
& \leq c|||u| \|_{s, b}^{3}
\end{aligned}
$$


where the last inequality follows from the trilinear estimates. Above, we also used the fact that $\mid 1-\psi(\tau-$ $\gamma(\xi)) \mid \leq 1$, and the fact that the $\xi, \tau$ integration is over the region $\left\{\xi, \tau \in \mathbb{R}^{2}:|\tau-\gamma(\xi)| \geq \frac{1}{2}\right\}$, since $|1-\psi(\tau-\gamma(\xi))|$ is 0 otherwise. Over this region,

$$
\frac{1}{|\tau-\gamma(\xi)|^{2}} \lesssim \frac{1}{\langle\tau-\gamma(\xi)\rangle^{2}}
$$

Proof of Lemma 2. We begin by taking the space-time Fourier transform of $\psi(t) I(x, t)$, and applying the $X_{s, b}$ norm. Using Fubini's theorem, we get

$$
\|\left.|\psi(t) I(x, t)|\right|_{s, b} ^{2} \simeq \int_{\mathbb{R}}\langle\xi\rangle^{2 s} \int_{\mathbb{R}}\left|\int_{\mathbb{R}}\langle\tau-\gamma(\xi)\rangle^{b} \widehat{\psi}(\lambda) \widehat{I}(\xi, \tau-\lambda) d \lambda\right|^{2} d \tau d \xi .
$$

Here, we use the fact that $\langle\tau-\gamma(\xi)\rangle \leq\langle\tau-\lambda-\gamma(\xi)\rangle+|\lambda| \leq\langle\tau-\lambda-\gamma(\xi)\rangle\langle\lambda\rangle$, along with Minkowski's inequality to get

$$
\left.|||\psi(t) I(x, t)|\right|_{s, b} ^{2} \leq \int_{\mathbb{R}}\langle\xi\rangle^{2 s}\left(\int_{\mathbb{R}}\langle\lambda\rangle^{b}|\widehat{\psi}(\lambda)|\left(\int_{\mathbb{R}}\langle\tau-\lambda-\gamma(\xi)\rangle^{2 b}|\widehat{I}(\xi, \tau-\lambda)|^{2} d \tau\right)^{\frac{1}{2}} d \lambda\right)^{2} d \xi .
$$

Which, after substituting $\tilde{\tau}=\tau-\lambda$, yields

$$
\begin{aligned}
\||\psi(t) I(x, t)|\|_{s, b}^{2} & \leq \int_{\mathbb{R}}\langle\xi\rangle^{2 s}\left(\int_{\mathbb{R}}\langle\tilde{\tau}-\gamma(\xi)\rangle^{2 b}|\widehat{I}(\xi, \tilde{\tau})|^{2} d \tilde{\tau}\right) \cdot\left(\int_{\mathbb{R}}\langle\lambda\rangle^{b}|\widehat{\psi}(\lambda)| d \lambda\right)^{2} d \xi \\
& \simeq c_{\psi}|||I(x, t)| \|_{s, b}^{2} .
\end{aligned}
$$

Estimate of (27). Let

$$
I(x, t) \doteq \frac{1}{2 \pi} \psi(t) \int_{\mathbb{R}} e^{i(\xi x+t \gamma(\xi))} c(\xi) d \xi
$$

where

$$
c(\xi) \doteq \int_{\mathbb{R}} \frac{1-\psi(\tau-\gamma(\xi))}{\tau-\gamma(\xi)} \widehat{w}_{u \bar{u} u}(\xi, \tau) d \tau .
$$

Taking the $X_{s, b}$ norm of $I$ yields

$$
\begin{aligned}
\||| I \mid\|_{s, b} & =\left(\iint_{\mathbb{R}^{2}}\langle\xi\rangle^{2 s}\langle\tau-\gamma(\xi)\rangle^{2 b}|c(\xi) \widehat{\psi}(\tau-\gamma(\xi))|^{2} d \xi d \tau\right)^{\frac{1}{2}} \\
& \lesssim c_{\psi}\left(\int_{\mathbb{R}}\langle\xi\rangle^{2 s}\left|\int_{\mathbb{R}} \frac{1-\psi(\tau-\gamma(\xi))}{\tau-\gamma(\xi)} \widehat{w}_{u \bar{u} u}(\xi, \tau) d \tau\right|^{2} d \xi\right)^{\frac{1}{2}} .
\end{aligned}
$$

Since $|\tau-\gamma(\xi)| \geq \frac{1}{2}$ and for $|x| \geq \frac{1}{2}, \frac{1}{|x|} \lesssim \frac{1}{\langle x\rangle}$,

$$
\begin{aligned}
\||I|\|_{s, b} & \lesssim c_{\psi}\left(\int_{\mathbb{R}}\langle\xi\rangle^{2 s}\left(\int_{|\tau-\gamma(\xi)| \geq \frac{1}{2}} \frac{\left|\widehat{w}_{u \bar{u} u}(\xi, \tau)\right|}{|\tau-\gamma(\xi)|} d \tau\right)^{2} d \xi\right)^{\frac{1}{2}}, \\
& \lesssim c_{\psi}\left(\int_{\mathbb{R}}\langle\xi\rangle^{2 s}\left(\int_{\mathbb{R}} \frac{\left|\widehat{w}_{u \bar{u} u}(\xi, \tau)\right|}{\langle\tau-\gamma(\xi)\rangle} d \tau\right)^{2} d \xi\right)^{\frac{1}{2}} .
\end{aligned}
$$

This, along with the trilinear estimate and the following lemma, is sufficient to show that $\||(27)| \mid\|_{s, b} \leq$ $c_{\psi}|||u|||_{s, b}^{3}$. 
Lemma 3. If $b>1 / 2$, then

$$
c_{\psi}\left(\int_{\mathbb{R}}\langle\xi\rangle^{2 s}\left(\int_{\mathbb{R}} \frac{\left|\widehat{w}_{u \bar{u} u}(\xi, \tau)\right|}{\langle\tau-\gamma(\xi)\rangle} d \tau\right)^{2} d \xi\right)^{\frac{1}{2}} \lesssim\left\||| w_{u \bar{u} u} \mid\right\|_{s, b-1} .
$$

Proof of Lemma 3. We follow the ideas of [16]. Should the reader desire more details, we refer them also to [17] and [18]. We see that by the Cauchy-Schwarz inequality, for any measurable function $I$,

$$
\begin{aligned}
\int_{\mathbb{R}}|I(\tau)| d \tau & =\int_{\mathbb{R}}\langle\tau-\gamma(\xi)\rangle^{-b}\langle\tau-\gamma(\xi)\rangle^{b}|I(\tau)| d \tau, \\
& \leq\left(\int_{\mathbb{R}}\langle\tau-\gamma(\xi)\rangle^{-2 b} d \tau\right)^{\frac{1}{2}}\left(\int_{\mathbb{R}}\langle\tau-\gamma(\xi)\rangle^{2 b}|I(\tau)|^{2} d \tau\right)^{\frac{1}{2}}, \\
& \stackrel{b>1 / 2}{\simeq}\left(\int_{\mathbb{R}}\langle\tau-\gamma(\xi)\rangle^{2 b}|I(\tau)|^{2} d \tau\right)^{\frac{1}{2}} .
\end{aligned}
$$

We can therefore conclude

$$
c_{\psi}\left(\int_{\mathbb{R}}\langle\xi\rangle^{2 s}\left(\int_{\mathbb{R}} \frac{\left|\widehat{w}_{u \bar{u} u}(\xi, \tau)\right|}{\langle\tau-\gamma(\xi)\rangle} d \tau\right)^{2} d \xi\right)^{\frac{1}{2}} \lesssim c_{\psi}||\left|w_{u \bar{u} u}\right| \|_{s, b}
$$

Estimate of (28). We will expand $e^{i t(\tau-\gamma(\xi))}-1$ into its power series and divide by $\tau-\gamma(\xi)$ to get

$$
\begin{aligned}
(28) & \simeq \psi(t) \iint_{\mathbb{R}^{2}} e^{i(\xi x+t \gamma(\xi))} \psi(\tau-\gamma(\xi)) \sum_{n=1}^{\infty} \frac{i^{n} t^{n}}{n !}(\tau-\gamma(\xi))^{n-1} \widehat{w}_{u \bar{u} u}(\xi, \tau) d \tau d \xi, \\
& \simeq \sum_{n=1}^{\infty} \frac{i^{n}}{n !} I_{n}(x, t),
\end{aligned}
$$

where

$$
\begin{aligned}
I_{n}(x, t) & \doteq \frac{1}{2 \pi} t^{n} \psi(t) \int_{\mathbb{R}} e^{i(\xi x+t \gamma(\xi))} c_{n}(\xi) d \xi \\
c_{n}(\xi) & \doteq \int_{\mathbb{R}} \psi(\tau-\gamma(\xi))(\tau-\gamma(\xi))^{n-1} \widehat{w}_{u \bar{u} u}(\xi, \tau) d \tau .
\end{aligned}
$$

It follows from the space-time Fourier transform of $I_{n}$, that

$$
\begin{aligned}
\||(31)|\|_{s, b} & \lesssim \sum_{n=1}^{\infty} \frac{1}{n !}\left\|\left|I_{n}(x, t)\right|\right\|_{s, b} \\
& =\sum_{n=1}^{\infty} \frac{1}{n !}\left(\iint_{\mathbb{R}^{2}}\langle\xi\rangle^{2 s}\langle\tau-\gamma(\xi)\rangle^{2 b}\left|c_{n}(\xi){\widehat{t^{n} \psi(t)}}^{t}(\tau-\gamma(\xi))\right|^{2} d \xi d \tau\right)^{\frac{1}{2}}, \\
& =\sum_{n=1}^{\infty} \frac{1}{n !}\left(\int_{\mathbb{R}}\langle\xi\rangle^{2 s}\left|c_{n}(\xi)\right|^{2}\left(\int_{\mathbb{R}}\left\langle\tau^{\prime}\right\rangle^{2 b}\left|{\widehat{t^{n} \psi(t)}}^{t}\left(\tau^{\prime}\right)\right|^{2} d \tau^{\prime}\right) d \xi\right)^{\frac{1}{2}}
\end{aligned}
$$

For all $b$ between 0 and 1 ,

$$
\left.\int_{\mathbb{R}}\left\langle\tau^{\prime}\right\rangle^{2 b}{\widehat{t^{n} \psi(t)}}^{t}\left(\tau^{\prime}\right)\right|^{2} d \tau^{\prime} \lesssim \int_{\mathbb{R}}\left\langle\tau^{\prime}\right\rangle^{2}\left|{\widehat{t^{n} \psi(t)}}^{t}\left(\tau^{\prime}\right)\right|^{2} d \tau^{\prime}=\left\|t^{n} \psi\right\|_{H^{1}}^{2}
$$

To deal with the $\left\|t^{n} \psi\right\|_{H^{1}}^{2}$ term, we take the derivative with respect to $t$ of $t^{n} \psi$ yielding

$$
\left\|t^{n} \psi\right\|_{H^{1}}^{2}=\left\|t^{n} \psi\right\|_{L^{2}}^{2}+\left\|n t^{n-1} \psi\right\|_{L^{2}}^{2}+\left\|t^{n} \psi^{\prime}\right\|_{L^{2}}^{2} \lesssim n^{2},
$$


since $\psi(t) \in C_{0}^{\infty}(-1,1)$. Thus

$$
\begin{aligned}
\|||(31) \mid\|_{s, b} & \lesssim \sum_{n=1}^{\infty} \frac{1}{n !}\left(\int_{\mathbb{R}}\langle\xi\rangle^{2 s}\left|c_{n}(\xi)\right|^{2}\left(\int_{\mathbb{R}}\left\langle\tau^{\prime}\right\rangle^{2 b}\left|{\widehat{t^{n} \psi(t)}}^{t}\left(\tau^{\prime}\right)\right|^{2} d \tau^{\prime}\right) d \xi\right)^{\frac{1}{2}}, \\
& \lesssim \sum_{n=1}^{\infty} \frac{n}{n !}\left(\int_{\mathbb{R}}\langle\xi\rangle^{2 s}\left|\int_{\tau \in \mathbb{R}} \psi(\tau-\gamma(\xi))(\tau-\gamma(\xi))^{n-1} \widehat{w}_{u \bar{u} u}(\xi, \tau) d \tau\right|^{2} d \xi\right)^{\frac{1}{2}} .
\end{aligned}
$$

Since the $\tau$-integration is over $|\tau-\gamma(\xi)| \leq 1$ and $\psi(\tau-\gamma(\xi)) \leq 1$,

$$
\begin{aligned}
\|\mid(31)\| \|_{s, b} & \lesssim\left(\int_{\mathbb{R}}\langle\xi\rangle^{2 s}\left(\int_{|\tau-\gamma(\xi)| \leq 1}\left|\widehat{w}_{u \bar{u} u}(\xi, \tau)\right| d \tau\right)^{2} d \xi\right)^{\frac{1}{2}} \cdot \sum_{n=1}^{\infty} \frac{n}{n !} \\
& \lesssim\left(\int_{\mathbb{R}}\langle\xi\rangle^{2 s}\left(\int_{\mathbb{R}} \frac{\left|\widehat{w}_{u \bar{u} u}(\xi, \tau)\right|}{\langle\tau-\gamma(\xi)\rangle} d \tau\right)^{2} d \xi\right)^{\frac{1}{2}} \\
& \lesssim\left\||| w_{u \bar{u} u} \mid\right\|_{s, b-1} \\
& \leq c \mid\|u\|_{s, b}^{3} .
\end{aligned}
$$

Combining these estimates concludes the proof of Proposition 1, which leaves only the proof of the trilinear estimates in order to prove Theorem 1.

\section{Proof of the Trilinear Estimates}

In this section, we prove the trilinear estimates presented in Theorem 2. The inequality (11) follows from [19], so we will focus on (12) and (13).

We will follow Tao's $[k ; Z]$-multiplier norm method developed in [26]. For the convenience of the reader, we provide an outline of the method. Let $Z$ be an abelian, additive group, with an invariant measure $d \xi$. For any $k \in \mathbb{N}, k \geq 2, \Gamma_{k}(Z)$ denotes the hyperplane

$$
\Gamma_{k}(Z):=\left\{\left(\xi_{1}, \ldots, \xi_{k}\right) \in Z: \xi_{1}+. .+\xi_{k}=0\right\},
$$

with the measure

$$
\int_{\Gamma_{k}(Z)} f:=\int_{Z^{k-1}} f\left(\xi_{1}, \ldots, \xi_{k-1},-\xi_{1} \ldots-\xi_{k-1}\right) d \xi_{1} \ldots d \xi_{k-1} .
$$

We define a $[k ; Z]$ - multiplier to be a function $m: \Gamma_{k}(Z) \rightarrow \mathbb{C}$, and the multiplier norm $\|m(\xi)\|_{[k ; Z]}$ is defined as the best constant such that the inequality

$$
\left|\int_{\Gamma_{k}(Z)} m(\xi) \prod_{j=1}^{k} f_{j}\left(\xi_{j}\right)\right| \leq\|m(\xi)\|_{[k ; Z]} \prod_{j=1}^{k}\left\|f_{j}\right\|_{L^{2}(Z)}
$$

holds for all test functions $f_{j}$ on $\mathrm{Z}$.

Summations over variables of the form $N_{j}, L_{j}$, and $H$ are presumed to be dyadic. Letting $N_{1}, N_{2}, N_{3}>0$, we will define the notation $N_{\max } \geq N_{\text {med }} \geq N_{\min }$ to be the maximum, medium, and minimum values of $N_{1}, N_{2}, N_{3}$. We similarly define $L_{\max } \geq L_{\text {med }} \geq L_{\text {min }}$. Summations over $L_{\max } \sim \ldots$ are sums over the dyadic variables $L_{1}, L_{2}, L_{3} \gtrsim 1$, that is,

$$
\sum_{L_{\max } \sim H}:=\sum_{L_{1}, L_{2}, L_{3} \gtrsim 1: L_{\max } \sim H} .
$$

Similarly, a sum of the form $N_{\max } \sim \ldots$ represents a sum over the variables $N_{1}, N_{2}, N_{3}>0$, that is,

$$
\sum_{N_{\text {max }} \sim N_{\text {med }} \sim N}:=\sum_{N_{1}, N_{2}, N_{3}>0: N_{\text {max }} \sim N_{\text {med }} \sim N} .
$$

Taking $\Gamma_{3}(\mathbb{R} \times \mathbb{R})$, where the hyperplane is parameterized by $(\xi, \tau)$, we are concerned with the resonance functions in the $(++-)$ case, $h_{1}(\xi)=h_{2}(\xi)=\xi^{2}-\xi^{3}$, and $h_{3}(\xi)=-\xi^{2}-\xi^{3}$. We define, for each $\mathrm{j}, \lambda_{j}=$ 
$\tau_{j}-h_{j}\left(\xi_{j}\right)$, and our resonance function $h(\xi)=h_{1}\left(\xi_{2}\right)+h_{2}\left(\xi_{2}\right)+h_{3}\left(\xi_{3}\right)=\xi_{1}^{2}+\xi_{2}^{2}-\xi_{3}^{2}-\xi_{1}^{3}-\xi_{2}^{3}-\xi_{3}^{3}$. Now, we have $\xi_{1}+\xi_{2}+\xi_{3}=0$, and $\lambda_{1}+\lambda_{2}+\lambda_{3}+h(\xi)=0$. When we do the dyadic decomposition, we will have $\left|\xi_{j}\right| \sim N_{j},\left|\lambda_{j}\right| \sim L_{j}$, and $|h(\xi)| \sim H$. Now, we have $\xi_{1}+\xi_{2}+\xi_{3}=0$, and $\lambda_{1}+\lambda_{2}+\lambda_{3}+h(\xi)=0$, which shows that

$$
N_{\text {max }} \sim N_{\text {med }} \gtrsim 1
$$

and

$$
L_{\text {max }} \sim \max \left(L_{\text {med }}, H\right) .
$$

Examining our resonance function, we can see that

$$
h(\xi)=\xi_{1}^{2}+\xi_{2}^{2}-\xi_{3}^{2}-\xi_{1}^{3}-\xi_{2}^{3}-\xi_{3}^{3}=-2 \xi_{1} \xi_{2}-3 \xi_{1} \xi_{2} \xi_{3}=-\xi_{1} \xi_{2}\left(2+3 \xi_{3}\right) .
$$

Which, for $\xi_{3} \neq \frac{-2}{3}$, allows us to show

$$
\max \left\{\lambda_{1}, \lambda_{2}, \lambda_{3}\right\} \geq \frac{1}{3}\left|\lambda_{1}+\lambda_{2}+\lambda_{3}\right| \gtrsim N_{1} N_{2} N_{3}
$$

With this in mind, we may assume $H \sim N_{\max }^{2} N_{\min }$, as the symbol vanishes otherwise. The issue now comes down to attempting to bound estimates of the form

$$
\left\|\left|X_{N_{1}, N_{2}, N_{2} ; H ; L_{1}, L_{2}, L_{3}}\right|\right\|_{[3, \mathbb{R} \times \mathbb{R}]},
$$

where $X_{N_{1}, N_{2}, N_{2} ; H ; L_{1}, L_{2}, L_{3}}$ is defined as

$$
X_{N_{1}, N_{2}, N_{2} ; H ; L_{1}, L_{2}, L_{3}}:=\chi_{|h(\xi)| \sim H} \prod_{j=1}^{3} \chi_{\left|\xi_{j}\right| \sim N_{j}} \chi_{\left|\lambda_{j}\right| \sim L_{j}} \cdot
$$

Lemma 4. Let $H, N_{1}, N_{2}, N_{3}, L_{1}, L_{2}, L_{3}>0$ satisfy (35) and (36), then

- ((++) coherence) If $N_{\max } \sim N_{\min }$, and $L_{\max } \sim H$, then we have

$$
(37) \lesssim L_{\text {min }}^{\frac{1}{2}} L_{\text {med }}^{\frac{1}{2}} N_{\text {max }}^{-1}
$$

- ((+-) coherence) If $N_{2} \sim N_{3} \gg N_{1}$, and $H \sim L_{1} \gtrsim L_{2}, L_{3}$, then

$$
(37) \lesssim L_{\min }^{\frac{1}{2}} N_{\max }^{-1} \min \left(H, \frac{N_{\max }}{N_{\min }} L_{\text {med }}\right)^{\frac{1}{2}},
$$

and similarly for permutations.

- In all other cases, we have

$$
(37) \lesssim L_{\text {min }}^{\frac{1}{2}} N_{\text {max }}^{-1} \min \left(H, L_{\text {med }}\right)^{\frac{1}{2}} .
$$

Proof. In the high modulation case, we have, and $L_{\max } \sim L_{\min } \gg H$. From [26], we can show that

$$
(37) \lesssim L_{\text {min }}^{\frac{1}{2}} N_{\text {min }}^{\frac{1}{2}} \lesssim L_{\text {min }}^{\frac{1}{2}} N_{\text {max }}^{-1} N_{\text {min }}^{\frac{1}{2}} N_{\text {max }} \simeq L_{\text {min }}^{\frac{1}{2}} N_{\text {max }}^{-1} H^{\frac{1}{2}} .
$$

In the low modulation cases, we have $L_{\max } \sim H$, here we must consider several cases, that is, (i) $L_{1} \geq L_{2} \geq L_{3}$, (ii) $L_{1} \geq L_{3} \geq L_{2}$, and (iii) $L_{3} \geq L_{2} \geq L_{1}$, the other cases follow by symmetry and permutation on the (12) indices.

In (i), by Corollary 4.2 in [26], we get, for $N_{1}, N_{2}, N_{3}>0$,

$$
(37) \lesssim L_{3}^{\frac{1}{2}}\left|\left\{\xi_{2} \in \mathbb{R}:\left|\xi_{2}-\xi_{2}^{0}\right| \ll N_{\min } ; h_{2}\left(\xi_{2}\right)+h_{3}\left(\xi-\xi_{2}\right)=\tau+\mathcal{O}\left(L_{2}\right)\right\}\right|^{\frac{1}{2}},
$$

for $\xi_{1}^{0}, \xi_{2}^{0},\left|\xi_{3}^{0}\right|$ satisfying $\left|\xi_{j}^{0}\right| \sim N_{j},\left|\xi_{1}^{0}+\xi_{2}^{0}+\xi_{3}^{0}\right| \ll N_{\text {min }}$, and for some $\tau, \xi \in \mathbb{R}$ satisfying $\left|\xi+\xi_{1}^{0}\right| \ll N_{\text {min }}$. We observe $h_{2}\left(\xi_{2}\right)+h_{3}\left(\xi-\xi_{2}\right)=h_{3}(\xi)+3 \xi_{2}\left(\xi-\xi_{2}\right)+2 \xi \xi_{2}=\tau+\mathcal{O}\left(L_{2}\right)$. Now, there are 3 subcases to 
consider: (1) $N_{1} \sim N_{2} \sim N_{3}$, (2) $N_{1} \sim N_{2} \gg N_{3}$, and (3) $N_{2} \sim N_{3} \gg N_{1}$, with the case (4) $N_{1} \sim N_{3} \gg N_{2}$ following by symmetry:

(1) In case (1) we can see that the $\xi_{2}$ variable is contained in an interval of length $\mathcal{O}\left(L_{2} N_{\max }^{-2}\right)$, so

$$
(37) \lesssim L_{\min }^{\frac{1}{2}} L_{\operatorname{med}}^{\frac{1}{2}} N_{\max }^{-1} .
$$

(2) In case 2, we similarly see that the $\xi_{2}$ variable is contained in an interval of length $\mathcal{O}\left(L_{2} N_{\max }^{-2}\right)$, so

$$
(37) \lesssim L_{\min }^{\frac{1}{2}} L_{\operatorname{med}}^{\frac{1}{2}} N_{\max }^{-1} .
$$

(3) In case (3), it follows that the $\xi_{2}$ variable is contained in an interval of length $\mathcal{O}\left(L_{2} N_{\max }^{-1} N_{\min }^{-1}\right)$, so

$$
(37) \lesssim L_{\text {min }}^{\frac{1}{2}} L_{\text {med }}^{\frac{1}{2}} N_{m a x}^{-\frac{1}{2}} N_{m i n}^{-\frac{1}{2}}
$$

and $\xi_{2}$ is also contained in an interval of length $\ll N_{\min }$. (ii) In (ii), by Corollary 4.2 in [26], we get, for $N_{1}, N_{2}, N_{3}>0$,

$$
(37) \lesssim L_{2}^{\frac{1}{2}}\left|\left\{\xi_{3} \in \mathbb{R}:\left|\xi_{3}-\xi_{3}^{0}\right| \ll N_{\text {min }} ; h_{3}\left(\xi_{3}\right)+h_{2}\left(\xi-\xi_{3}\right)=\tau+\mathcal{O}\left(L_{3}\right)\right\}\right|^{\frac{1}{2}}
$$

for $\xi_{1}^{0}, \xi_{2}^{0},\left|\xi_{3}^{0}\right|$ satisfying $\left|\xi_{j}^{0}\right| \sim N_{j},\left|\xi_{1}^{0}+\xi_{2}^{0}+\xi_{3}^{0}\right| \ll N_{\text {min }}$, and for some $\tau, \xi \in \mathbb{R}$ satisfying $\left|\xi+\xi_{1}^{0}\right| \ll$ $N_{\text {min }}$. We now observe $h_{3}\left(\xi_{3}\right)+h_{2}\left(\xi-\xi_{3}\right)=h_{2}(\xi)-2 \xi \xi_{3}+3 \xi_{3}\left(\xi-\xi_{3}\right)=\tau+\mathcal{O}\left(L_{3}\right)$. In similar subcases to before, for subcase (1), we again obtain

$$
(37) \lesssim L_{\min }^{\frac{1}{2}} L_{\text {med }}^{\frac{1}{2}} N_{\max }^{-1}
$$

in subcase (2) ((3) follows here by symmetry), we get

$$
(37) \lesssim L_{\text {min }}^{\frac{1}{2}} L_{\text {med }}^{\frac{1}{2}} N_{\max }^{-\frac{1}{2}} N_{\min }^{-\frac{1}{2}}
$$

and in subcase (4),

$$
(37) \lesssim L_{\text {min }}^{\frac{1}{2}} L_{\text {med }}^{\frac{1}{2}} N_{\text {max }}^{-1}
$$

Finally, in (iii), again by Corollary 4.2 in [26], we get, for $N_{1}, N_{2}, N_{3}>0$,

$$
(37) \lesssim L_{2}^{\frac{1}{2}}\left|\left\{\xi_{2} \in \mathbb{R}:\left|\xi_{2}-\xi_{2}^{0}\right| \ll N_{\min } ; h_{2}\left(\xi_{2}\right)+h_{1}\left(\xi-\xi_{2}\right)=\tau+\mathcal{O}\left(L_{2}\right)\right\}\right|^{\frac{1}{2}}
$$

for $\xi_{1}^{0}, \xi_{2}^{0},\left|\xi_{3}^{0}\right|$ satisfying $\left|\xi_{j}^{0}\right| \sim N_{j},\left|\xi_{1}^{0}+\xi_{2}^{0}+\xi_{3}^{0}\right| \ll N_{\text {min }}$, and for some $\tau, \xi \in \mathbb{R}$ satisfying $\left|\xi+\xi_{3}^{0}\right| \ll$ $N_{\text {min }}$. We now observe $h_{2}\left(\xi_{2}\right)+h_{1}\left(\xi-\xi_{2}\right)=h_{2}(\xi)+\left(\xi-\xi_{2}\right)\left(3 \xi_{2}-2 \xi_{2}\right)=\tau+\mathcal{O}\left(L_{2}\right)$. Again, in subcases (1) and (3), we get

$$
(37) \lesssim L_{\text {min }}^{\frac{1}{2}} L_{\text {med }}^{\frac{1}{2}} N_{\text {max }}^{-1}
$$

and in subcases (2) and (4), we are left with

$$
(37) \lesssim L_{\min }^{\frac{1}{2}} L_{\operatorname{med}}^{\frac{1}{2}} N_{\max }^{-\frac{1}{2}} N_{\min }^{-\frac{1}{2}}
$$

Now, we will apply this to our proof of the the trilinear estimates. For (12), we have $\|\left|(u \bar{u} u)_{x}\right|||_{s, b-1} \lesssim$ $\||| u \mid\|_{s, b}^{3}$. By duality and Plancharel, we must show that

$$
\left\|\frac{\left(\xi_{1}+\xi_{2}+\xi_{3}\right)\left\langle\xi_{4}\right\rangle^{s}}{\left\langle\tau_{4}+\xi_{4}^{2}+\xi_{4}^{3}\right\rangle^{1-b}\left\langle\tau_{2}+\xi_{2}^{2}+\xi_{2}^{3}\right\rangle^{b}\left\langle\tau_{1}-\xi_{1}^{2}+\xi_{1}^{3}\right\rangle^{b}\left\langle\tau_{3}-\xi_{3}^{2}+\xi_{3}^{3}\right\rangle^{b} \prod_{j=1}^{3}\left\langle\xi_{j}\right\rangle^{s}}\right\|_{[4, \mathbb{R} \times \mathbb{R}]} \lesssim 1 .
$$


We estimate $\left|\xi_{1}+\xi_{2}+\xi_{3}\right|$ by $\left\langle\xi_{4}\right\rangle$, then use the fractional Leibniz rule to show

$$
\left\langle\xi_{4}\right\rangle^{s+1} \lesssim\left\langle\xi_{4}\right\rangle^{\frac{1}{2}} \sum_{j=1}^{3}\left\langle\xi_{j}\right\rangle^{s+\frac{1}{2}}
$$

By symmetry of the 1 and 3 indices, it remains to prove

$$
\left\|\frac{\left\langle\xi_{1}\right\rangle^{\frac{1}{2}}\left\langle\xi_{2}\right\rangle^{-s}\left\langle\xi_{4}\right\rangle^{\frac{1}{2}}\left\langle\xi_{3}\right\rangle^{-s}}{\left\langle\tau_{4}+\xi_{4}^{2}+\xi_{4}^{3}\right\rangle^{1-b}\left\langle\tau_{2}+\xi_{2}^{2}+\xi_{2}^{3}\right\rangle^{b}\left\langle\tau_{1}-\xi_{1}^{2}+\xi_{1}^{3}\right\rangle^{b}\left\langle\tau_{3}-\xi_{3}^{2}+\xi_{3}^{3}\right\rangle^{b}}\right\| \|_{[4, \mathbb{R} \times \mathbb{R}]} \lesssim 1
$$

and,

$$
\left.\left\|\frac{\left\langle\xi_{1}\right\rangle^{-s}\left\langle\xi_{2}\right\rangle^{\frac{1}{2}}\left\langle\xi_{4}\right\rangle^{\frac{1}{2}}\left\langle\xi_{3}\right\rangle^{-s}}{\left\langle\tau_{4}+\xi_{4}^{2}+\xi_{4}^{3}\right\rangle^{1-b}\left\langle\tau_{2}+\xi_{2}^{2}+\xi_{2}^{3}\right\rangle^{b}\left\langle\tau_{1}-\xi_{1}^{2}+\xi_{1}^{3}\right\rangle^{b}\left\langle\tau_{3}-\xi_{3}^{2}+\xi_{3}^{3}\right\rangle^{b}}\right\|\right|_{[4, \mathbb{R} \times \mathbb{R}]} \lesssim 1 .
$$

By minorizing $\left\langle\tau_{1}-\xi_{1}^{2}+\xi_{1}^{3}\right\rangle^{b}$ by $\left\langle\tau_{1}-\xi_{1}^{2}+\xi_{1}^{3}\right\rangle^{1-b}$ in (40), and $\left\langle\tau_{2}+\xi_{2}^{2}+\xi_{2}^{3}\right\rangle^{b}$ by $\left\langle\tau_{2}+\xi_{2}^{2}+\xi_{2}^{3}\right\rangle^{1-b}$ in (41), then applying conjugation/TT* identity, (40) reduces to the bilinear estimates

$$
\|u \bar{v}\|_{L^{2}} \lesssim\|u\|\left\|_{X^{-1 / 2,1-b}} \mid\right\| v\|\|_{X^{s, b}}
$$

and

$$
\left.\|\bar{u} v\|_{L^{2}} \lesssim\|u\|\right|_{X^{-1 / 2,1-b}}\left|\left\|v|\||_{X^{s, b}} .\right.\right.
$$

For (13), we seek to show that $\left\|\left|\partial_{x}(u \mathcal{H}(u \bar{u}))\right|||_{s, b-1} \lesssim\right\| \| u||_{s, b}^{3}$. By duality and Plancharel, it suffices to show

$$
\left\|\frac{\operatorname{sign}\left(\xi_{1}+\xi_{2}\right)\left(\xi_{1}+\xi_{2}+\xi_{3}\right)\left\langle\xi_{4}\right\rangle^{s}}{\left\langle\tau_{4}+\xi_{4}^{2}+\xi_{4}^{3}\right\rangle^{1-b}\left\langle\tau_{2}+\xi_{2}^{2}+\xi_{2}^{3}\right\rangle^{b}\left\langle\tau_{1}-\xi_{1}^{2}+\xi_{1}^{3}\right\rangle^{b}\left\langle\tau_{3}-\xi_{3}^{2}+\xi_{3}^{3}\right\rangle^{b} \prod_{j=1}^{3}\left\langle\xi_{j}\right\rangle^{s}}\right\|_{[4, \mathbb{R} \times \mathbb{R}]} \lesssim 1 .
$$

But $(42) \lesssim(40)$, so the result follows from the previous discussion.

Proof of (42). By Plancharel, it suffices to show that

$$
\left\|\frac{\left\langle\xi_{1}\right\rangle^{-s}\left\langle\xi_{2}\right\rangle^{\frac{1}{2}}}{\left\langle\tau_{2}+\xi_{2}^{2}+\xi_{2}^{3}\right\rangle^{1-b}\left\langle\tau_{1}-\xi_{1}^{2}+\xi_{1}^{3}\right\rangle^{b}}\right\|_{[3, \mathbb{R} \times \mathbb{R}]}
$$

Following from [26], we reduce this to showing that

$$
\sum_{N_{\max } \sim N_{\text {med }} \sim N} \sum_{L_{1}, L_{2}, L_{3} \gtrsim 1} \frac{\left\langle N_{1}\right\rangle^{-s}\left\langle N_{2}\right\rangle^{\frac{1}{2}}}{L_{1}^{b} L_{2}^{1-b}}\left\|X_{N_{1}, N_{2}, N_{2} ; L_{\max } ; L_{1}, L_{2}, L_{3}}\right\|_{[3, \mathbb{R} \times \mathbb{R}]} \lesssim 1,
$$

and,

$$
\sum_{N_{\text {max }} \sim N_{\text {med }} \sim N} \sum_{L_{\max } \sim L_{\text {med }}} \sum_{H \ll L_{\max }} \frac{\left\langle N_{1}\right\rangle^{-s}\left\langle N_{2}\right\rangle^{\frac{1}{2}}}{L_{1}^{b} L_{2}^{1-b}}\left\|X_{N_{1}, N_{2}, N_{2} ; H ; L_{1}, L_{2}, L_{3}}\right\|_{[3, \mathbb{R} \times \mathbb{R}]} \lesssim 1 .
$$

We first prove (44). Because $H \ll L_{\text {max }} \sim L_{\text {med }}$, from (39), $\left\|X_{N_{1}, N_{2}, N_{2} ; H ; L_{1}, L_{2}, L_{3}}\right\|_{[3, \mathbb{R} \times \mathbb{R}]} \lesssim L_{\text {min }}^{\frac{1}{2}} N_{\text {max }}^{-1} H^{\frac{1}{2}}$. Plugging this in, (44) becomes

$$
\sum_{N_{\text {max }} \sim N_{\text {med }} \sim N} \sum_{L_{\text {max }} \sim L_{\text {med }} \gtrsim N_{1} N_{2} N_{3}} \frac{\left\langle N_{1}\right\rangle^{-s}\left\langle N_{2}\right\rangle^{\frac{1}{2}}}{L_{1}^{b} L_{2}^{1-b}} L_{\text {min }}^{\frac{1}{2}} N_{\text {max }}^{-1}\left(N_{1} N_{2} N_{3}\right)^{\frac{1}{2}} \lesssim 1 .
$$

Tao dealt with a similar term in [26], following his method, we estimate $\frac{\left\langle N_{2} \frac{1}{2}\right\rangle}{\left\langle N_{1}{ }^{s}\right\rangle} \lesssim \frac{N^{\frac{1}{2}}}{\left\langle N_{\min }\right\rangle^{s}}$, and $L_{1}^{b} L_{2}^{1-b} \gtrsim$ $L_{\text {min }}^{b} L_{\text {med }}^{1-b}$. Performing the L summation yields

$$
\sum_{N_{\max } \sim N_{\text {med }} \sim N} \frac{N^{\frac{1}{2}}\left(N^{2} N_{\min }\right)^{b-1 / 2}}{\left\langle N_{\min }\right\rangle^{s} N} \lesssim 1
$$


which holds for $s>-1 / 4$, and $1 / 2<b<3 / 4$.

Proof of (43). First, we deal with the case $N_{1} \sim N_{2} \sim N_{3} \sim N \gtrsim 1$. In this case, (37) holds, $L_{\max } \sim N^{3}$, so (43) reduces to showing

$$
\sum_{L_{\max } \sim N^{3}} \frac{N^{\frac{1}{2}}}{N^{s} L_{\min }^{b} L_{\text {med }}^{1-b}} L_{\min }^{\frac{1}{2}} L_{\text {med }}^{\frac{1}{2}} N_{\max }^{-1} \lesssim 1
$$

Which holds for $s>1 / 4$. Finally, we have three remaining cases. (i) $N \sim N_{1} \sim N_{2} \gg N_{3} ; H \sim L_{3} \gtrsim L_{1}, L_{2}$, (ii) $N \sim N_{2} \sim N_{3} \gg N_{1} ; H \sim L_{1} \gtrsim L_{2}, L_{3}$, and (iii) $N \sim N_{1} \sim N_{3} \gg N_{2} ; H \sim L_{2} \gtrsim L_{1}, L_{3}$.

In (i), it reduces to

$$
\sum_{N_{3} \ll N} \sum_{1 \lesssim L_{1}, L_{2} \lesssim N^{2} N_{3}} \frac{N^{\frac{1}{2}}}{N^{s} L_{1}^{b} L_{2}^{1-b}} L_{\min }^{\frac{1}{2}} N^{-1} \min \left(N^{2} N_{3}, \frac{N}{N_{3}} L_{m e d}\right)^{\frac{1}{2}} \lesssim 1
$$

Performing the outer summation yields

$$
\sum_{1 \lesssim L_{1}, L_{2} \lesssim N^{3}} \frac{N^{1 / 2-1+3 / 4-s}}{L_{1}^{b} L_{2}^{1-b}} L_{\min }^{\frac{1}{2}} L_{\text {med }}^{\frac{1}{4}} \lesssim 1,
$$

which holds for $s>1 / 4$, and $1 / 2<b<3 / 4$. To get both (ii) and (iii), we minorize $L_{1}^{b}$ by $L_{1}^{1-b}$, it suffices to show

$$
\sum_{N_{1} \ll N} \sum_{1 \lesssim L_{1}, L_{2} \ll N^{2} N_{1}} \frac{N^{\frac{1}{2}}}{\left\langle N_{1}\right\rangle^{s}\left(N^{2} N_{1}\right)^{1-b} L_{2}^{1-b}} L_{\min }^{\frac{1}{2}} N_{1}^{\frac{1}{2}} \lesssim 1 .
$$

Assuming $N_{1} \gtrsim N^{-2}$, since the L sum vanishes otherwise, we can perform the L summation to yield,

$$
\sum_{N^{-2} \lesssim N_{1} \ll N} \frac{N^{\frac{1}{2}} N_{1}^{\frac{1}{2}}}{\left\langle N_{1}\right\rangle^{S}\left(N^{2} N_{1}\right)^{3 / 2-2 b}} \lesssim 1,
$$

which holds for $s>1 / 2$, and $1 / 2<b<3 / 4$. Finally, we must deal with the case where (39) holds. We reduce to showing

$$
\sum_{N_{\text {max }} \sim N_{\text {med }} \sim N} \sum_{L_{\text {max }} \sim N_{1} N_{2} N_{3}} \frac{\left\langle N_{2}\right\rangle^{\frac{1}{2}}}{\left\langle N_{1}\right\rangle^{s} L_{1}^{b} L_{2}^{1-b}} L_{\text {min }}^{\frac{1}{2}} N^{-1} L_{\text {med }}^{\frac{1}{2}} \lesssim 1 .
$$

Doing the L summations, we reduce to showing

$$
\sum_{N_{\max } \sim N_{\text {med }} \sim N} \frac{\left\langle N_{2}\right\rangle^{\frac{1}{2}}\left(N_{1} N_{2} N_{3}\right)^{b-1 / 2}}{\left\langle N_{1}\right\rangle^{s}} N^{-1} \lesssim 1,
$$

which holds for $s>-1 / 2$, and $1 / 2<b<3 / 4$.

The proof of (42) follows from a similar estimate with the roles of $L_{1}$ and $L_{2}$ reversed.

Author Contributions: All authors contributed equally to the writing of this paper. All authors read and approved the final manuscript.

Conflicts of Interest: "The authors declare no conflict of interest."

\section{References}

[1] Cousins, W., \& Sapsis, T. (2016). Reduced-order precursors of rare events in unidirectional nonlinear water waves. Journal of Fluid Mechanics, 790, 368-388.

[2] Mosincat, R., Pilod, D., \& Saut, J. C. (2021). Global well-posedness and scattering for the Dysthe equation in $L^{2}\left(\mathcal{R}^{2}\right)$. Journal de Mathèmatiques Pures et Appliquées, 149, 73-97.

[3] Dysthe, K. B., \& Trulsen, K. (1999). Note on breather type solutions of the NLS as models for freak-waves. Physica Scripta, 1999(T82), 48.

[4] Onorato, M., Osborne, A. R., Serio, M., \& Cavaleri, L. (2005). Modulational instability and non-Gaussian statistics in experimental random water-wave trains. Physics of Fluids, 17(7), 078101. 
[5] Akhmediev, N., \& Pelinovsky, E. (2010). Editorial-introductory remarks on "discussion \& debate: Rogue waves-towards a unifying concept?". The European Physical Journal Special Topics, 185(1), 1-4.

[6] Chabchoub, A., Hoffmann, N., Onorato, M., \& Akhmediev, N. (2012). Super rogue waves: observation of a higher-order breather in water waves. Physical Review X, 2(1), 011015.

[7] Chabchoub, A., Hoffmann, N. P., \& Akhmediev, N. (2011). Rogue wave observation in a water wave tank. Physical Review Letters, 106(20), 204502.

[8] Cousins, W., \& Sapsis, T. P. (2014). Quantification and prediction of extreme events in a one-dimensional nonlinear dispersive wave model. Physica D: Nonlinear Phenomena, 280, 48-58.

[9] Goullet, A., \& Choi, W. (2011). A numerical and experimental study on the nonlinear evolution of long-crested irregular waves. Physics of Fluids, 23(1), 016601.

[10] Lo, E., \& Mei, C. C. (1985). A numerical study of water-wave modulation based on a higher-order nonlinear Schrödinger equation. Journal of Fluid Mechanics, 150, 395-416.

[11] Dyachenko, A. I., \& Zakharov, V. E. E. (2011). Compact equation for gravity waves on deep water. JETP Letters, 93(12), 701-705.

[12] Fedele, F. (2014). On certain properties of the compact Zakharov equation. Journal of Fluid Mechanics, 748, $692-711$.

[13] Bourgain, J. (1993). Fourier transform restriction phenomena for certain lattice subsets and applications to nonlinear evolution equations Part I. Geometric E Functional Analysis, 3(2), 107-156.

[14] Bourgain, J. (1993). Fourier transform restriction phenomena for certain lattice subsets and applications to nonlinear evolution equations Part II. Geometric E Functional Analysis, 3(3), 209-262.

[15] Colliander, J., Keel, M., Staffilani, G., Takaoka, H., \& Tao, T. (2002). Almost conservation laws and global rough solutions to a nonlinear Schrödinger equation. Mathematical Research Letters 9, 659-682.

[16] Kenig, C. E., Ponce, G., \& Vega, L. (1996). A bilinear estimate with applications to the KdV equation. Journal of the American Mathematical Society, 573-603.

[17] Gorsky, J., \& Himonas, A. A. (2009). Well-posedness of KdV with higher dispersion. Mathematics and Computers in Simulation, 80(1), 173-183.

[18] Gorsky, J., Himonas, A. A., Holliman, C., \& Petronilho, G. (2013). The Cauchy problem of a periodic higher order KdV equation in analytic Gevrey spaces. Journal of Mathematical Analysis and Applications, 405(2), 349-361.

[19] Carvajal, X. (2004). Local well-posedness for a higher order nonlinear Schrödinger equation in Sobolev spaces of negative indices. Electronic Journal of Differential Equations, 2004(13), 1-10.

[20] Fokas, A., Himonas, A., \& Mantzavinos, D. (2017). The nonlinear Schrödinger equation on the half-line. Transactions of the American Mathematical Society, 369(1), 681-709.

[21] Hannah, H., Himonas, A. A., \& Petronilho, G. (2011). Gevrey regularity of the periodic gKdV equation. Journal of Differential Equations, 250(5), 2581-2600.

[22] Himonas, A. A., \& Petronilho, G. (2012). Analytic well-posedness of periodic gKdV. Journal of Differential Equations, 253(11), 3101-3112.

[23] Kenig, C. E., Ponce, G., \& Vega, L. (2001). On the ill-posedness of some canonical dispersive equations. Duke Mathematical Journal, 106(3), 617-633.

[24] Staffilani, G. (1997). On the generalized Korteweg-de Vries-type equations. Differential and Integral Equations, 10(4), 777-796.

[25] Takaoka, H. (1999). Well-posedness for the one-dimensional nonlinear Schrödinger equation with the derivative nonlinearity. Advances in Differential Equations, 4(4), 561-580.

[26] Tao, T. (2001). Multilinear weighted convolution of $L^{2}$ functions, and applications to nonlinear dispersive equations. American Journal of Mathematics, 123(5), 839-908.

[27] Tao, T. (2006). Nonlinear Dispersive Equations: Local and Global Analysis (No. 106). American Mathematical Society.

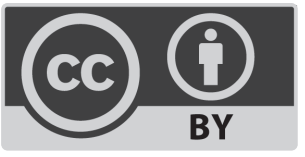

(C) 2021 by the authors; licensee PSRP, Lahore, Pakistan. This article is an open access article distributed under the terms and conditions of the Creative Commons Attribution (CC-BY) license (http://creativecommons.org/licenses/by/4.0/). 